\section{UJMM

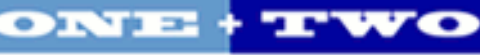

Volume 10 | 2020 Spring 2020

\section{Undergraduate Journal of Mathematical}

Modeling: One + Two

\title{
Finding the Growth Rate of a Tumor
}

Christine Staat

University of South Florida

Advisors:

Arcadii Grinshpan, Mathematics and Statistics

Laurie Staat, Moffit Cancer Hospital

Problem Suggested By: Christine Staat

Follow this and additional works at: https://digitalcommons.usf.edu/ujmm

Part of the Mathematics Commons

UJMM is an open access journal, free to authors and readers, and relies on your support:

Donate Now

\section{Recommended Citation}

Staat, Christine (2020) "Finding the Growth Rate of a Tumor," Undergraduate Journal of Mathematical Modeling: One + Two: Vol. 10: Iss. 2, Article 5.

DOI: https://doi.org/10.5038/2326-3652.10.2.4918

Available at: https://digitalcommons.usf.edu/ujmm/vol10/iss2/5 


\title{
Finding the Growth Rate of a Tumor
}

\begin{abstract}
The Gompertz method is used to analyze the growing glioblastoma data and estimate how accurate the results of growth over time are. The Gompertz curve is expressed as $V(t)=\alpha e^{-\beta e^{-\gamma t}}$. The data from the tumor is graphed in Excel along with the values from the Gompertz equation. Excel solver is used to assist in determining the constant values of $\alpha, \beta$, and $\gamma$. The data of the tumor is overall very close to the outcome of the Gompertz model following a sigmoidal " $S$ " curve.
\end{abstract}

\section{Keywords}

tumor, glioblastoma, Gompertz method, Gompertz differential equation, Gompertz curve

Creative Commons License

(c) $(1) \Theta \Theta$

This work is licensed under a Creative Commons Attribution-Noncommercial-Share Alike 4.0 License. 


\section{PROBLEM STATEMENT}

Apply Gompertz method with data from a tumor's development over seventy days to compare the accuracy of the method to real life results and to reveal the tumor's maximum volume of growth.

\section{MOTIVATION}
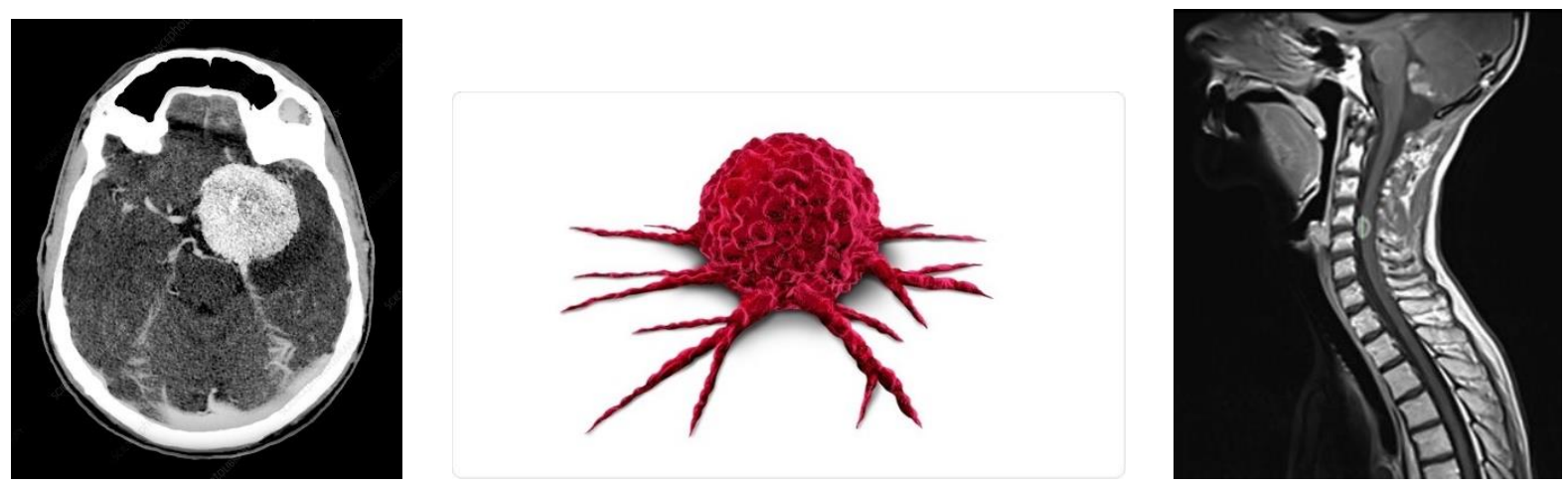

Cancer is a major threat to public health in today's world. It is the leading cause of death in humanity and many animals. Scientists have done an immense amount of research on how cancers grow, thrive and expand but they have not found an ultimate way to cure this disease, especially since cancers can mutate by changing their DNA to adapt to harmful environments.

As tumors grow, they start to advance within a body, working on conquering an area to use for their benefit. At any moment they can divide cells from a mass to travel into other parts of the body, slowly but steadily invading a person from the inside out. A strategy that has been found to be favorable is to observe how tumors grow and to develop methods to measure them. This is very significant in furthering research to better understand how cancer develops. Being able to transform and redesign itself in small amounts of time to further infect its host, cancer is very elusive to detect and eradicate. So far, many treatment therapies such as radiation and chemotherapy to work on defeating this war against cancer have been found. Nonetheless, cancer affects everyone differently and it also evolves quickly, building resistance against the methodologies that are currently available.

The pace of the tumor's growth is a very important information for doctors, in order to evaluate how aggressive the cancer could be and to administer the best plan of action for the patient. The growth rate of the tumor greatly depends on how it is treated, along with where it is located. If it is possible, doctors prefer to remove malignant masses immediately to prevent the mutated cells from spreading. To understand the precise growth of each tumor, scientists have introduced a growth rate equation to measure the volume of cancer cells over a certain time period. This information gives us an idea on how the tumor will behave so doctors can strategize a strong treatment plan before the tumor has any time to react. 
There are a variety of differential growth models to choose from. In this paper, we have decided to utilize the Gompertz Model, due to its simplicity and accuracy. Benjamin Gompertz originally created this model in 1825 to help explain human mortality rates (Murphy, Jaafari, \& Dobrovolny, 2016). Makeham first specified to use this model in its most cumulative form, to find the best curve using only log-transformed values to determine sums of squares. This method was used until the 1940's then Hartley proposed and first explained how to linearize the Gompertz equation (Tjørve, 2017). When the Gompertz equation was linearized and suitably integrated, it has become what we use today. Being very popular Gompertz method is used for many different fields such as demands of certain goods and products, growth in traffic, and biological growth of animals. The Gompertz method is based on a differential equation that is used to determine growth rates for many different subject areas. It is exceptionally valuable to help explain tumor dynamics.

\section{MATHEMATICAL DESCRIPTION AND SOLUTION APPROACH}

The Gompertz method is used to show the development of a tumor as a function of time, in terms of its volume. It is based on an exponential formula that gives values that are fairly accurate to how the tumor will behave. With these reliable approximations, doctors are able to formulate meticulous plans for treating various cancers. The classical Gompertz differential equation is:

$$
\frac{d V}{d t}=\alpha(\ln \beta-\ln V) V
$$

where $V$ is volume in cubic millimeters, $t$ represents time, and $\alpha$ and $\beta$ are constants (Stewart \& Day, p.504). Its general solution is:

$$
V(t)=\alpha e^{-\beta e^{-\gamma t}}
$$

The volume $V$ cannot be equal 0 nor can $\beta$ be equal to $V$. If this were the case, then the tumor's rate of growth would be 0 .

Equation (2) uses information that correlates with a tumor's existing condition. The values are usually portrayed by a sigmoidal curve which appears as an " $\mathrm{S}$ " shape showing slow growth at first, followed by an exponential climb and then it plateaus with its slowest growth towards the end. This equation involves three parameters: alpha $\alpha$ is the asymptote of the graph which we find by setting the limit of $t$ to infinity $\left(\lim _{\mathrm{t} \rightarrow \infty} V(\mathrm{t})=\lim _{\mathrm{t} \rightarrow \infty} \alpha e^{-\beta e^{-\gamma t}}\right)$, beta $\beta$ is the displacement of time on the $x$-axis, and gamma $\gamma$ is the volumetric growth rate. These values are usually retrieved or calculated from a given data set. 


\section{DATA ANALYSIS AND RESUlTS}

For the average patient, data on tumor growth is usually recorded only once or twice because MRI and CT scans are very expensive. For research purposes, scientists have done a handle full of experiments where a tumor is scanned on a regular basis to gain knowledge on the growth rate and behavior of certain types of cancer. The field advisor of this research project has retrieved data from one of the experiments that focused on the development of a glioblastoma. This is a type of brain tumor that grows quickly and is very aggressive.

The information gathered on this tumor is the volumetric size over a seventy-day period, shown in Table 1. Growth in cubic millimeters is graphed on the y-axis and time in days is graphed on the $x$-axis. The information is put into Excel to observe the shape of graph produced which creates a "S" shape curve.

The next step is to find the constant values, $\alpha, \beta$, and $\gamma$, for the Gompertz model. Since the given information on the tumor is very limited, estimated guesses are made to find close values to what the constants could be. Gompertz equation is also inputted into Excel using the constants and each set of data points. From there, a residual column is made in effort to help determine the values of the constants. This column is considered as our error margin and is the difference between the y value and our Gompertz value. The residual value is then squared with the total sum of the values entered into the box labeled SSR (sum of squared residuals). Then we insert the value of the SSR into solver (in Excel) to assist in finding the best value for each constant. Solver works on adjusting the SSR value to minimize the error as much as possible which changes the constant values to help fit the best numbers into the equation according to our data. The constant values are included in Table 1. The values of the Gompertz method are then plotted on to the same graph with the tumor data to compare how the method matches the actual values. This can be viewed on Graph 1. 
Table 1.

\begin{tabular}{|c|c|c|c|c|}
\hline X-Time (Days & )Y- Size (Volume $\mathrm{mm}^{\wedge}$ & 3)Gompertz- $\mathrm{V}(\mathrm{t})=\alpha \mathrm{EXP}$ & )/Residual & Residual $^{2}$ \\
\hline 1 & 151 & 140.7456663 & 10.25433368 & 105.1513593 \\
\hline 5 & 178 & 188.0911076 & -10.09110763 & 101.8304532 \\
\hline 10 & 226 & 257.4204142 & -31.42041423 & 987.2424307 \\
\hline 15 & 329 & 336.2714541 & -7.271454083 & 52.87404448 \\
\hline 20 & 433 & 422.1937301 & 10.80626991 & 116.7754694 \\
\hline 25 & 564 & 512.46607 & 51.53393005 & 2655.745946 \\
\hline 30 & 598 & 604.4039548 & -6.403954754 & 41.01063649 \\
\hline 35 & 687 & 695.588552 & -8.588552015 & 73.76322572 \\
\hline 40 & 796 & 784.0060141 & 11.99398587 & 143.8556971 \\
\hline 45 & 855 & 868.1059542 & -13.10595417 & 171.7660348 \\
\hline 50 & 934 & 946.7981643 & -12.79816428 & 163.793009 \\
\hline 55 & 1001 & 1019.408898 & -18.40889772 & 338.8875153 \\
\hline 60 & 1089 & 1085.615557 & 3.384442612 & 11.45445179 \\
\hline 65 & 1143 & 1145.37412 & -2.374119563 & 5.636443702 \\
\hline 70 & 1217 & 1198.848865 & 18.15113451 & 329.4636839 \\
\hline \multicolumn{5}{|l|}{ Constants } \\
\hline$\alpha$ & $\boldsymbol{\beta}$ & $\gamma$ & & SSR \\
\hline 1557.598923 & 2.482452936 & 0.032135485 & & 5299.2504 \\
\hline
\end{tabular}

Graph 1.

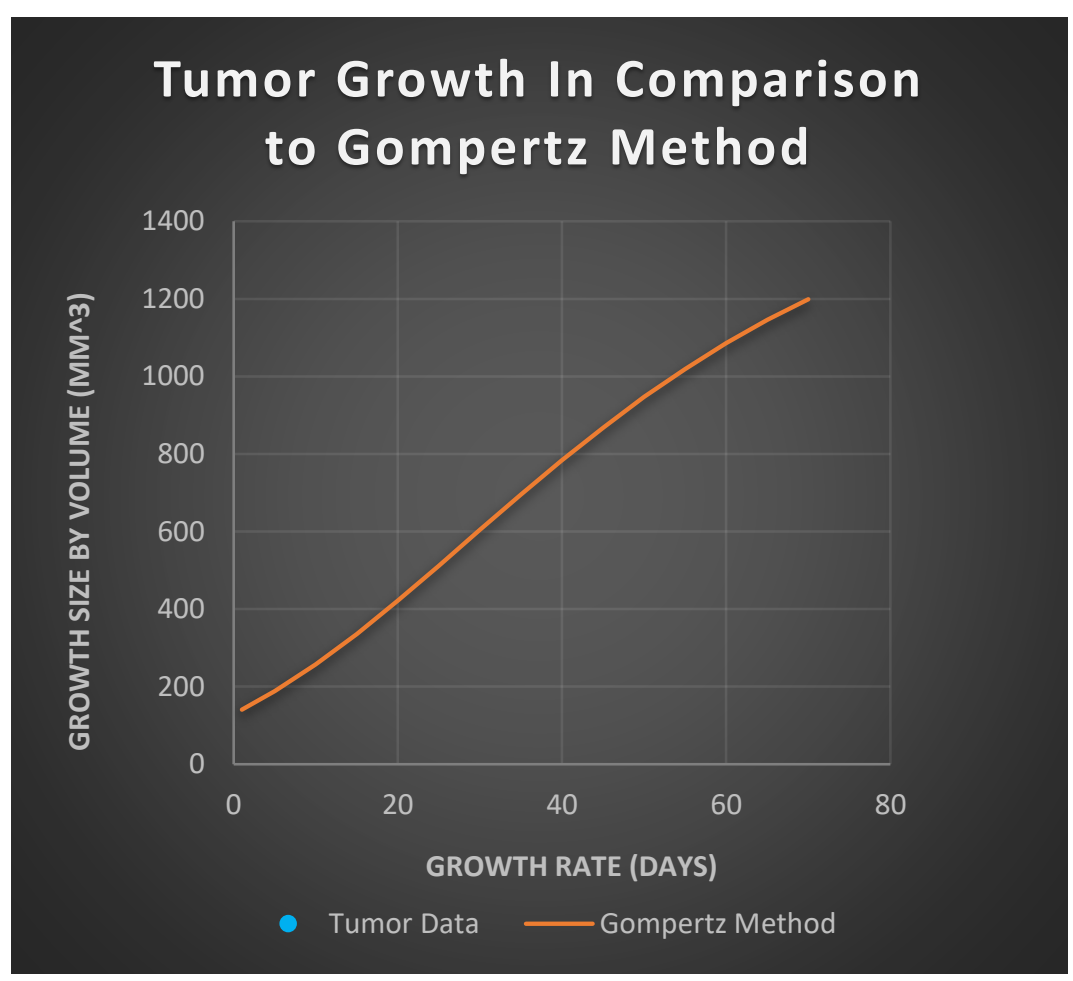

Therefore, the Gompertz model for this particular tumor, using equation 1, is given by:

$$
V(t)=1557.59 e^{-2.48 e^{-0.032 t}} \text {. }
$$




\section{CONCLUSION AND RECOMMENDATIONS}

The growth rate of this tumor is extreme, increasing by almost $10 \%$ in 70 days. Such an exponential growth rate means that there is much less time to prepare a treatment plan for the individual who is suffering from this disease. These aggressive tumors are ideal for this method because it allows us to foresee the development and behavior in order to prevent them from metastasizing. The final results show the Gompertz method to be a correct fit to estimate the exponential growth of a glioblastoma accurately. With this method we are able to find the maximum growth limit of the tumor, $\alpha$, to be 1557.59 and the constant growth rate, $\gamma$, to be 0.032 .

The objective of this project to determine the maximum volume for this tumor is accomplished. Also we successfully compared Gompertz method to tumor data to analyze if it would correlate in the same way as portraying a sigmoidal curve on a graph. It would be recommended to evaluate Gompertz method again with a larger data set and time frame. This method works very well for the available amount of information.

NOMENCLATURE

\begin{tabular}{|l|l|l|}
\hline$V$ & Volume & $\mathrm{mm}^{3}$ \\
\hline $\mathrm{t}$ & Time & Days \\
\hline$\alpha$ & Alpha- Growth Limit & $\mathrm{mm}^{3}$ \\
\hline$\beta$ & Beta- Rate of Time & Days \\
\hline$\gamma$ & Gamma- Growth Rate & $\mathrm{mm}^{3} /$ day \\
\hline
\end{tabular}




\section{REFERENCES}

Tjørve, K., \& Tjørve, E. (2017). The use of Gompertz models in growth analyses, and new Gompertz-model approach: An addition to the Unified-Richards family. PloS one, 12(6), e0178691. doi:10.1371/journal.pone.0178691

Stewart, J., \& Day, T. (2015). Biocalculus: Calculus, Probability, and Statistics for the Life Sciences. Cengage Learning, Boston MA.

Murphy, H., Jaafari, H. \& Dobrovolny, H.M.(2016). Differences in predictions of ODE models of tumor growth: a cautionary example. BMC Cancer 16, 163; doi:10.1186/s12885-016-2164-X 